\title{
Parallel fabrication and single-electron charging of devices based on ordered, two-dimensional phases of organically functionalized metal nanocrystals
}

\author{
Gil Markovich, Daniel V. Leff, and Sung-Wook Chung \\ Molecular Design Institute, Lawrence Berkeley Laboratory and Department of Chemistry and Biochemistry, \\ University of California at Los Angeles, Los Angeles, California 90095-1569 \\ Hermes M. Soyez and Bruce Dunn \\ Department of Materials Science and Engineering, University of California at Los Angeles, Los Angeles, \\ California 90024-1565 \\ James R. Heath ${ }^{\text {a) }}$ \\ Molecular Design Institute, Lawrence Berkeley Laboratory and Department of Chemistry and Biochemistry, \\ University of California at Los Angeles Los Angeles, California 90095-1569
}

(Received 3 January 1997; accepted for publication 9 April 1997)

A parallel technique for fabricating single-electron, solid-state capacitance devices from ordered, two-dimensional closest-packed phases of organically functionalized metal nanocrystals is presented. The nanocrystal phases were prepared as Langmuir monolayers and subsequently transferred onto Al-electrode patterned glass substrates for device construction. Alternating current impedance measurements were carried out to probe the single-electron charging characteristics of the devices under both ambient and $77 \mathrm{~K}$ conditions. Evidence of a Coulomb blockade and step structure reminiscent of a Coulomb staircase is presented. (C) 1997 American Institute of Physics. [S0003-6951(97)03123-9]

The study of solid-state devices based on single-electron tunneling has been an intense area of research over the last decade. ${ }^{1-3}$ One promising route to the fabrication of such devices is the use of nanometer-size metal and semiconductor particles as the active device elements. ${ }^{4-8}$ Solution-phase chemical methods for synthesizing these passivated metal $^{9,10}$ and semiconductor nanocrystals ${ }^{11,12}$ have rapidly evolved over the past few years. For single-electron device applications, recent work has indicated that passivated nanocrystals of coinage metals may be particularly useful. ${ }^{8}$ Also, singleelectron energy level spacings in metal particles are dominated by simple electrostatics, and may be readily tuned through the modification of nanocrystal size. For sufficiently small ( $\sim 2-4$-nm-diam) particles, the single-electron charging energies that characterize a particle are much greater than $\mathrm{kT}$ at room temperature, suggesting the possibility of operation of metal nanocrystal-based single-electron devices at ambient temperature.

A major challenge associated with incorporating nanometer-size particles into electronic devices in any parallel fabrication technique is related to the preparation of uniform thin films consisting of narrow size distributions of nanocrystals. Granular metal films and heteroepitaxially grown quantum dot islands are two possibilities, and indeed much important work has been done using these techniques. ${ }^{67,13,14}$ However, control over particle size, size distribution, and particle density, in general, is nontrivial ${ }^{15-17}$ especially for particles below $10 \mathrm{~nm}$ in diameter.

One alternative is to use a Langmuir trough to prepare a film of nanocrystals and to transfer that film to a substrate. ${ }^{18}$ This technique has certain advantages. Particle size and composition (i.e., the nature of the metal and the surface passivant) are selected prior to film deposition, and the film may

${ }^{\text {a) }}$ Author to whom correspondence should be addressed. be transferred to virtually any substrate of any size. We have recently shown that metal nanocrystals on a Langmuir trough may be compressed into any one of a number of phases, depending on the applied pressure and the chemical details of particle-particle interactions. ${ }^{16}$ Such broad control over the properties of nanocrystal thin films, coupled with electrical measurements, should provide a powerful route toward fabricating single-electron devices. Also, phenomena related to particle composition as well as interparticle interactions may be investigated.

In this letter, we report on a parallel technique for fabricating single-electron capacitance devices from a 2D closestpacked organically functionalized Ag nanocrystal layer. We also report on the results of ac impedance measurements ( $\Delta C-V$ characteristics) carried out to probe the singleelectron charging characteristics of the devices under both ambient and $77 \mathrm{~K}$ conditions. Evidence of a Coulomb blockade, a step structure reminiscent of a Coulomb staircase, and memory charging effects is presented.

The devices were fabricated in three distinct steps: (1) nanocrystal preparation and size selection, (2) preparation and substrate transfer of the nanocrystal Langmuir monolayer, and (3) device construction into a sandwich-type configuration. All particles used here were dodecanethiol-capped 3 -nm-diam $(\sigma<10 \%)$ Ag nanocrystals. The particle synthesis and the subsequent size selection using solvent-pair precipitation techniques are described in detail elsewhere. ${ }^{16,19,20}$ All particles were selectively precipitated up to four times, and were characterized by $\mathrm{x}$-ray powder diffraction and ultraviolet/visible (UV/vis) absorption spectroscopy. More extensive characterizations of these particles are described elsewhere. ${ }^{16,19,21}$

Langmuir monolayer film formation was performed as described by Heath et al. ${ }^{16}$ on a Nima Technology type 611 Langmuir trough at room temperature, with a $1 \mathrm{mg} / \mathrm{ml}$ solution of $\mathrm{Ag}$ nanocrystals in clean hexane. The $\mathrm{Ag}$ particles 


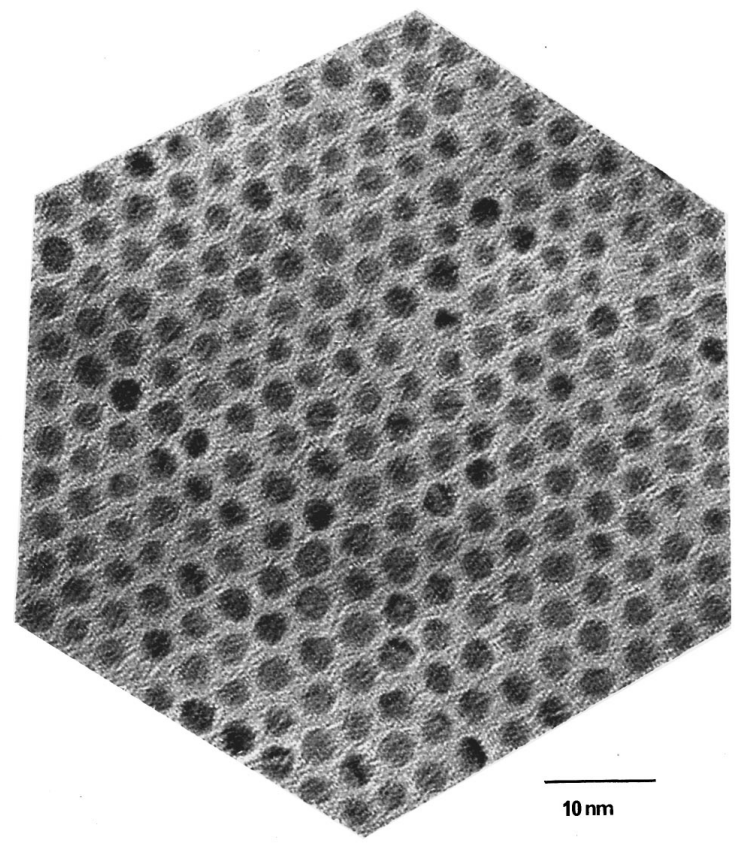

FIG. 1. TEM micrograph of a closest-packed phase of dodecanethiol-capped 3.0-nm-diam Ag nanocrystals prepared on a Langmuir trough and transferred to a TEM grid. This micrograph was cropped to highlight the crystallographic structure of the phase. The 2D crystalline domains extend up to $1 \mu \mathrm{m}$ or so in any given direction, but the particle density is continuous over the entire phase (many $\mathrm{cm}^{2}$ ).

were narrow size distribution "case II" particles, as described in Ref. 16, which means that they will compress into an ordered, hexagonal phase. A transmission electron microscopy (TEM) micrograph of a phase similar to those prepared in the present work is shown in Fig. 1. This micrograph was cropped to highlight the crystallographic structure of the phase. Ordered domains in these films typically extend over $0.1-1 \mu \mathrm{m}^{2}$, although the particle density is continuous over many square centimeters. For device fabrication, a film that had been compressed to just below the (2D)-(collapsed 2D) phase boundary was transferred as a LangmuirSchaeffer film ${ }^{16}$ to a glass substrate that had been prepatterned with a series of 1-mm-wide Al lines.

After the nanocrystal film was transferred to the substrate, the particles were cross-linked by exchanging their surface groups with 1, 10-decanedithiol. This procedure, similar to that described by Andres and coworkers, ${ }^{22}$ was necessary for stablilizing the film against damage during subsequent processing steps.

Once the nanocrystal film was stabilized, a $35 \mathrm{~nm}$ (measured by profilometry) dielectric spacer layer of polymethylmethacrylate (PMMA) was deposited by spin coating. Finally, a pattern of 1-mm-wide Al lines, oriented perpendicular to the bottom Al line pattern, was evaporated onto the PMMA layer. For this step, the substrate was kept at $77 \mathrm{~K}$ to prevent thermal damage to the underlying particle layer. Ultimately, the processed substrate contained many "active" devices arranged in a parallel fashion, the number of which is only limited by the density of the Al grid lines. A crosssectional view (not to scale) of a single device is shown in Fig. 2(A).

After device construction, two (one top and one bottom) $\mathrm{Al}$ electrodes were bonded to wires using silver paint, and
(A)

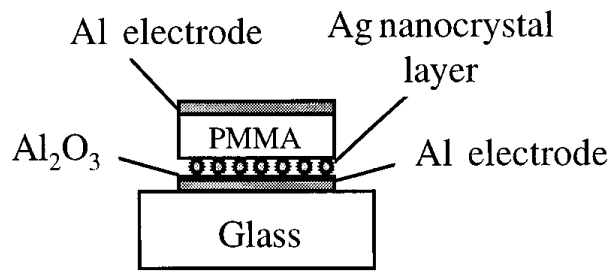

(B)

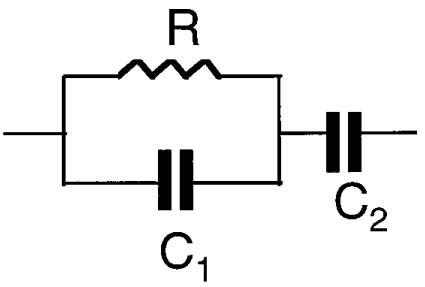

(C)

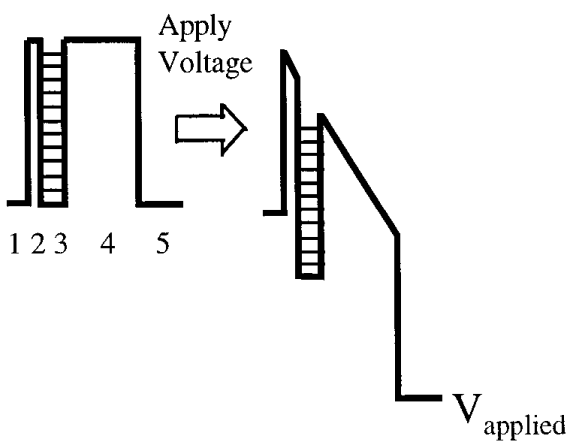

FIG. 2. (A) Cross-sectional schematic of a device (not to scale); (B) equivalent RC circuit; (C) equivalent energy-level diagram. In (B), $C_{1}$ represents the $\mathrm{Al} / \mathrm{Al}_{2} \mathrm{O}_{3}$ /nanocrystal junction capacitance, $R$ is the same junction's tunneling resistance, and $C_{2}$ represents the nanocrystal/PMMA/Al junction capacitance. In $(\mathrm{C})$, regions 1 and 5 are $\mathrm{Al}$ electrode layers, region 2 is an $\mathrm{Al}_{2} \mathrm{O}_{3}$ layer $(1 \mathrm{~nm})$, region 3 is the metal nanocrystal monolayer $(5 \mathrm{~nm})$, and region 4 is the PMMA insulating spacer layer $(30-40 \mathrm{~nm})$.

the wires were connected to the measuring circuit. The substrate was mounted on the cold finger of an immersion Dewar, and cooled to $77 \mathrm{~K}$. A voltage was applied across the circuit with the use of a function generator (generating a 30-500 $\mathrm{mV}_{p-p}$ amplitude sinusoidal wave) floated with a tunable dc offset. The ac response of the device, as a function of the dc offset, was recorded with a lock-in amplifier.

We consider the device to be a parallel array of double tunneling junctions. The resistance of the polymer layer is practically infinite and therefore we can represent the nanocrystals/polymer/aluminum junction as a pure capacitive element. To analyze the ac response of the device, it is useful to draw an equivalent RC circuit for the double junction [Fig. 2(B).$^{13}$ Analysis of this RC circuit shows that the capacitance measured as the off-phase component of the ac current through the device is approximately $C_{2}$ in our case.

Figure 2(C) represents the equivalent energy-level diagram of the capacitance device. As the voltage applied ( $\left.V_{\text {applied }}\right)$ between the two Al electrodes is varied, singleelectron energy levels of the particles are brought into resonance with the Fermi level of the nearby electrode. If the modulation is strong enough, electrons can tunnel back and forth between the Fermi level of the nearby electrode and the energy levels of the particles, thereby producing charge os- 


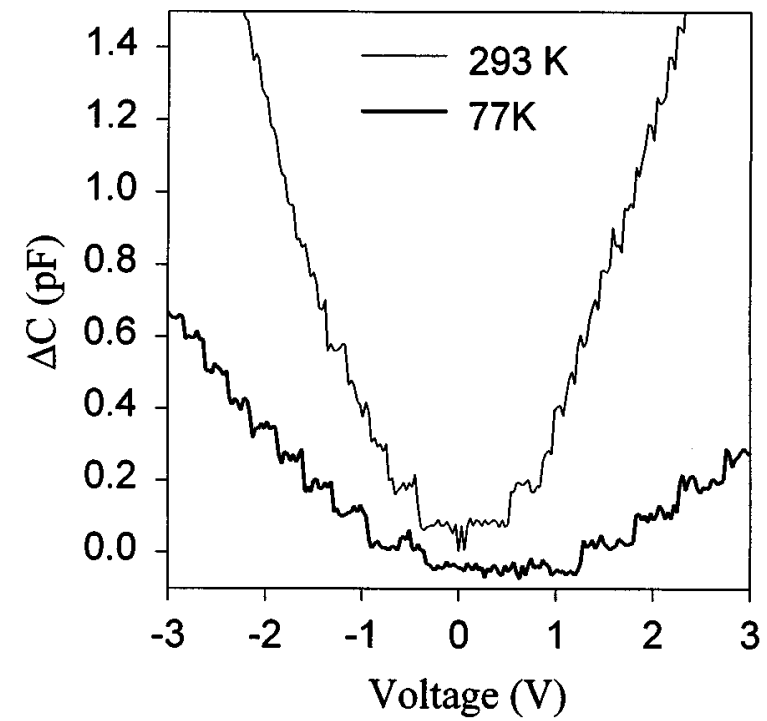

FIG. 3. Representative ac response measurements $(\Delta C-V$ characteristics) of a device probed at $77 \mathrm{~K}$ and ambient temperature. The change in capacitance $\left(\Delta C_{2}\right)$ as a function of applied voltage for a single 2 min scan is shown for each temperature. As can be seen, a Coulomb blockade and a step structure reminiscent of a Coulomb staircase are present. All of the devices tested $(\approx 20)$ exhibited similar responses.

cillations on the remote electrode and an increase in the capacitance signal.

In Fig. 3 we show representative ac impedance measurements $(\Delta C-V$ characteristics) of the device operated at 77 and $293 \mathrm{~K}$. A Coulomb blockade about zero bias (asymmetric) and a step structure reminiscent of a Coulomb staircase ${ }^{7}$ are visible in both capacitance/voltage curves; however, these features are more pronounced in the $77 \mathrm{~K}$ scan. Each curve consists of a single $(2 \mathrm{~min})$ voltage scan from positive to negative polarity of the bottom electrode. We believe that each of the steps reflects an increase in capacitance of the device due to collective single-electron (or hole) charging of the particles in the monolayer film. The step structure is reproducible from device to device over multiple scans and different frequencies.

The $\Delta C-V$ curves in Fig. 3 were taken with a relatively large amplitude modulation $\left(400 \mathrm{mV}_{p-p}\right)$. The step structure is still apparent at $77 \mathrm{~K}$ with $50 \mathrm{mV}_{p-p}$ modulation, although with lower signal-to-noise. From the step heights of both curves (about $0.1 \mathrm{pF}$ ), we estimate that $\sim 10^{6}$ particles, or $<1 \%$ of the particles in a given junction, are being charged in each step. Control experiments on devices containing no nanocrystals revealed no Coulomb blockade or staircase structure.

Deviations from the ideal behavior of a Coulomb blockade (symmetric structure about zero bias) and a Coulomb staircase (uniform step width, step height, and overall structure) are expected for these devices. Physical phenomena that may influence the single-electron charging behavior of the devices include (1) low-temperature memory effects, similar to those previously observed for granular metal systems; ${ }^{13,23}$ (2) electrostatic interactions between adjacent particles (particle center-to-center distances here are about 4-5 nm).

The simple parallel fabrication technique, discussed in this letter, may be readily extended to include not only other phases but semiconductor and other metal nanocrystals as well. Measurements similar to those reported here, coupled with control over nanocrystal film density, nanocrystal composition, and morphology of the nanocrystals within the film (e.g., rings, wires, extended closest-packed phases, etc.), should make it possible to probe discrete quantum energy levels. The influence of particle-particle interactions in nanocrystal-based solid-state devices may also be investigated. Efforts along these lines are currently underway.

This work was supported by the Office of Naval Research, Order No. N00014-95-F0099 and by the Director, Office of Energy Research, Office of Basic Energy Research, Division of Materials Sciences of the U.S. Department of Energy under Contract No. DE-AC03-76SF00098, and by the NSF-NYI Program. One author (J.R.H.) acknowledges support from the David and Lucile Packard Foundation, and a Dreyfus fellowship. A second author (G.M.) acknowledges support from the Fulbright Program. Two authors (B.D. and H.M.S.) acknowledge funding from the NSF (DMR9408780).

${ }^{1}$ M. A. Kastner, Phys. Today 46, 24 (1993).

${ }^{2}$ D. V. Averin and K. K. Likharev, Mesoscopic Phenomena in Solids, edited by B. L. Altshuler, P. A. Lee, and R. A. Webb (Elsevier, Amsterdam, 1991).

${ }^{3}$ D. Averin and K. K. Likharev, Single-Charge Tunneling, edited by H. Grabert and M. H. Devoret (Plenum, New York, 1992).

${ }^{4}$ V. L. Colvin, M. C. Schlamp, and A. P. Alivisatos, Nature (London) 370, 354 (1994).

${ }^{5}$ B. O. Dabbousi, M. G. Bawendi, O. Onitsuka, and M. F. Rubner, Appl. Phys. Lett. 66, 1316 (1995).

${ }^{6}$ K. H. Schmidt, G. Medeiros-Ribiero, M. Oestreich, P. M. Petroff, and G. H. Dohler, Phys. Rev. B 54, 11346 (1996).

${ }^{7}$ J. B. Barner and S. T. Ruggiero, Phys. Rev. Lett. 59, 807 (1987); R. Wilkins, E. Ben-Jacob, and R. C. Jaklevic, ibid. 63, 801 (1989).

${ }^{8}$ R. P. Andres, T. Bein, M. Dorogi, S. Feng, J. I. Henderson, C. P. Kubiak, W. Mahoney, R. G. Osifchin, and R. Reifenberger, Science 272, 1323 (1996).

${ }^{9}$ G. Schmid, Chem. Rev. 92, 1709 (1992).

${ }^{10}$ M. Brust, M. Walker, D. Bethell, D. J. Schiffrin, and R. Whyman, J. Chem. Soc. Chem. Commun. 801 (1994).

${ }^{11}$ M. G. Bawendi, M. L. Steigerwald, and L. E. Brus, Annu. Rev. Phys. Chem. 41, 477 (1990).

${ }^{12}$ A. P. Alivisatos, J. Phys. Chem. 100, 13226 (1996).

${ }^{13}$ J. G. A. Dubois, J. W. Gerritsen, G. Schmid, and H. van Kempen, Physica B 218, 262 (1996).

${ }^{14}$ D. L. Klein, P. L. McEuen, J. E. Bowen Katari, R. Roth, and A. P. Alivisatos, Appl. Phys. Lett. 68, 2574 (1996).

${ }^{15}$ D. D. Awschalom and D. P. DiVincenzo, Phys. Today 48, 43 (1995).

${ }^{16}$ L. N. Lewis, Chem. Rev. 93, 2693 (1993).

${ }^{17}$ J. Lambe and R. C. Jaklevic, Phys. Rev. Lett. 22, 1371 (1969).

${ }^{18}$ G. Medeiros-Ribeiro, D. Leonard, and P. M. Petroff, Appl. Phys. Lett. 66, 1767 (1995).

${ }^{19}$ P. C. Ohara, D. V. Leff, J. R. Heath, and W. M. Gelbart, Phys. Rev. Lett. 75, 3466 (1995).

${ }^{20}$ J. R. Heath, C. M. Knobler, and D. V. Leff, J. Phys. Chem. 101, 189 (1997).

${ }^{21}$ S. A. Harfenist, Z. L. Wang, M. M. Alvarez, I. Vezmar, and R. L. Whetten, J. Phys. Chem. 100, 13904 (1996).

${ }^{22}$ B. O. Dabbousi, C. B. Murray, M. F. Rubner, and M. G. Bawendi, Chem. Mater. 6, 216 (1994).

${ }^{23}$ D. V. Leff, P. C. Ohara, J. R. Heath, and W. M. Gelbart, J. Phys. Chem. 99, 7036 (1995).

${ }^{24}$ C. B. Murray, D. J. Norris, and M. G. Bawendi, J. Am. Chem. Soc. 115, 8706 (1993)

${ }^{25}$ D. V. Leff, L. Brandt, and J. R. Heath, Langmuir 12, 4723 (1996).

${ }^{26}$ R. P. Andres, J. D. Bielefeld, J. I. Henderson, D. B. Janes, V. R. Kolagunta, C. P. Kubiak, W. J. Mahoney, and R. G. Osifchin, Science 273, 1690 (1996)

${ }^{27}$ R. E. Cavicchi and R. H. Silsbee, Phys. Rev. B 38, 6407 (1988). 